\title{
Measurement of Polarized Drell-Yan Process at FNAL E1039/SpinQuest
}

\author{
PacificSpin2019 @ Miyazaki, Japan \\ 2019-August-29
}

Kenichi Nakano

Tokyo Tech

This manuscript has been authored by Fermi Research Alliance, LLC under Contract No. DEAC02-07CH11359 with the U.S. Department of Energy, Office of Science, Office of High Energy Physics. 


\section{Outline}

1. Introduction

$\triangleright$ Sivers function of anti-quarks

2. Measurement at E1039/SpinQuest

$\triangleright$ Measurement method

$\triangleright$ Anticipated sensitivity

3. Experimental status

$\triangleright$ Setups of target \& spectrometer

$\triangleright$ Schedule toward data taking

4. Summary 


\section{Sivers Function: $f_{1 T}^{\perp}\left(x, k_{T}\right)$}

- One of the eight Transverse-Momentum-Dependent (TMD) PDFs

\begin{tabular}{cc|ccc}
\hline & & \multicolumn{3}{|c}{ Parton spin } \\
\hline Nucleon & $\mathrm{U}$ & $\mathrm{U}$ & $\mathrm{L}$ & $\mathrm{T}$ \\
spin & $\mathrm{L}$ & & & Boer-Mulders $h_{1}^{\perp}$ \\
& $\mathrm{T}$ & Sivers $f_{1 T}^{\perp}$ & Helicity $g_{1}$ & Worm gear \#2 $h_{1 L}^{\perp}$ \\
& & & & Transversity $h_{1} \&$ \\
& & & Pretzelosity $h_{1 T}^{\perp}$ \\
\hline
\end{tabular}

- Correlation between the nucleon spin $(S) \&$ the parton transverse momentum $\left(k_{T}\right)$

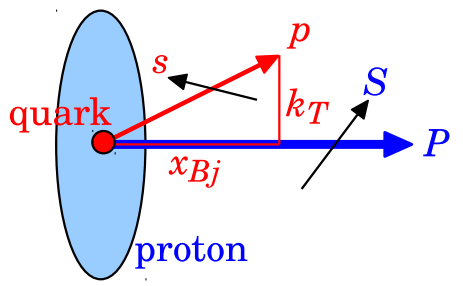




\section{Sivers Function of Anti-Quarks}

- Extraction by global analyses

$\triangleright$ PRD88 (2013) 114012, P. Sun \& F. Yuan

$\triangleright$ PRD89 (2014) 074013, M. G. Echevarria et at.

$\triangleright$ JHEP 04 (2017) 046, M. Anselmino et al.

$\triangle \triangleright$ Use of HERMES, COMPASS \& JLab data

$\triangleright \triangleright$ First moment of Sivers function:

$$
x \Delta^{N} f^{(1)}(x) \equiv-x f_{1 T}^{\perp(1)}(x)
$$

- $f_{1 T}^{\perp}(x)$ of anti-quarks is not well known

$\triangleright$ Since $\bar{q} \& q$ are mixed up in SIDIS

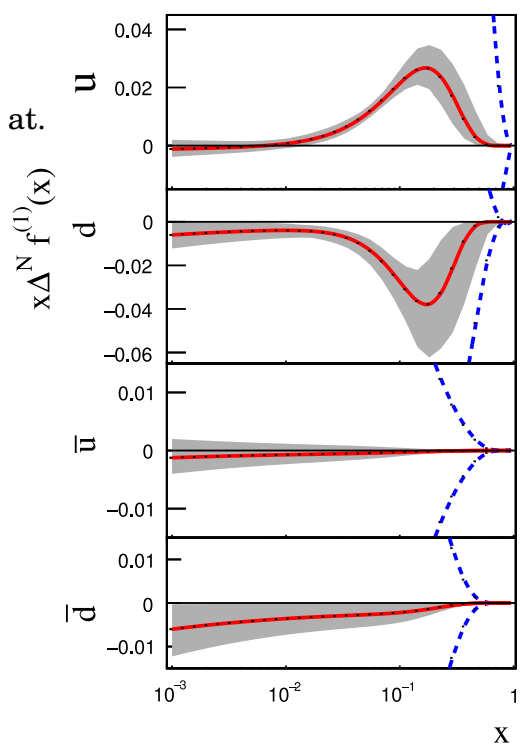




\section{Importance of Anti-Quarks}

In proton spin puzzle: $\frac{1}{2}=\frac{1}{2} \Delta \Sigma+J_{G}+L_{q}+L_{\bar{q}}$

Lattice QCD

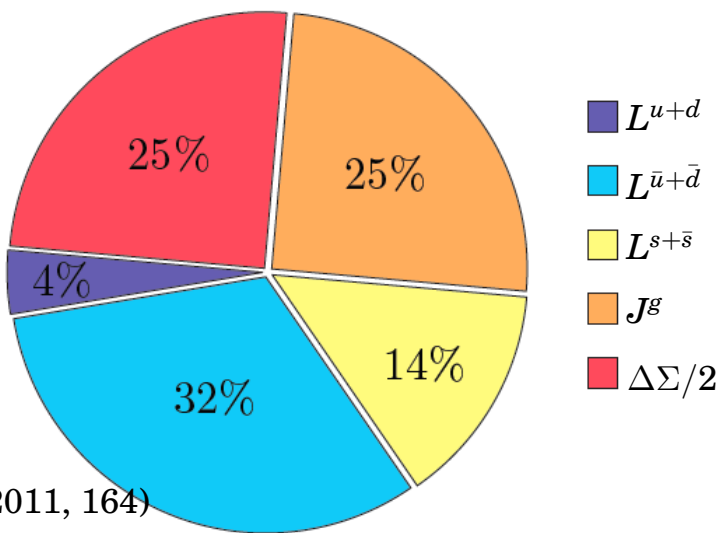

(PoS LATTICE2011, 164)

- The sea-quark orbital angular momentum is expected to be a major part of the missing spin

- Non-zero Sivers function of anti-quarks promises $L_{\bar{q}} \neq 0$ ! 


\section{Connection to Unpolarized PDF}

- Light anti-quark flavor asymmetry: $\bar{d}(x) / \bar{u}(x)$

$\triangleright$ E866/NuSea

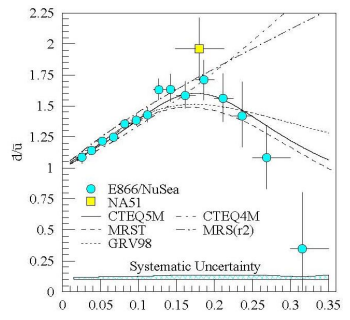

$\triangleright$ E906/SeaQuest
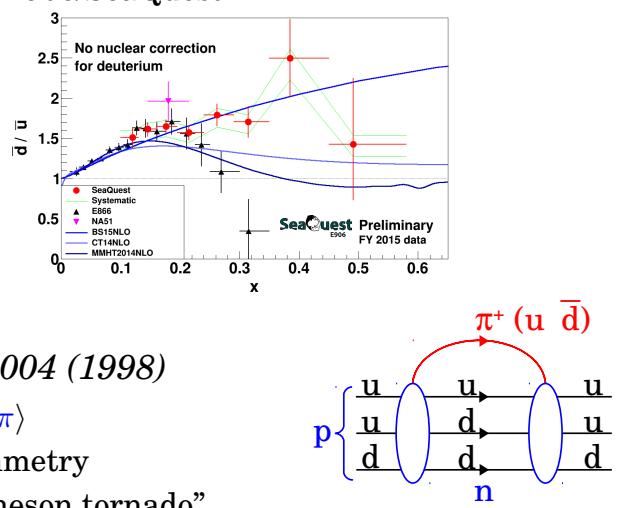

- Meson cloud model ... PRD58, 092004 (1998)

$\triangleright|p\rangle=(1-a-b)\left|p_{0}\right\rangle+a|N \pi\rangle+b|\Delta \pi\rangle$

$\triangleright$ It predicts the large $\bar{d}(x) / \bar{u}(x)$ asymmetry

$\triangleright$ It predicts also non-zero $L_{q, \bar{q}}$ like "meson tornado" (need $L=1$ of $\pi$ to make $J^{P}=1 / 2^{+}$of proton, as parity of $\pi$ is $J^{P}=0^{-}$)

- We could reach an unified understanding about the Sivers function, the OAM \& the $\bar{d}(x) / \bar{u}(x)$ asymmetry 


\section{Measurement at E1039/SpinQuest}




\section{Proton-Induced Drell-Yan Process}

- Cross section at LO

$$
\begin{aligned}
\frac{d^{2} \sigma}{d x_{\text {beam }} d x_{\text {target }}}= & \frac{4 \pi \alpha^{2}}{9 x_{\text {beam }} x_{\text {target }}} \frac{1}{s} \sum_{i=u, d, \cdots} e_{i}{ }^{2} . \\
& \left\{q_{i}\left(x_{\text {beam }}\right) \bar{q}_{i}\left(x_{\text {target }}\right)+\bar{q}_{i}\left(x_{\text {beam }}\right) q_{i}\left(x_{\text {target }}\right)\right\}
\end{aligned}
$$

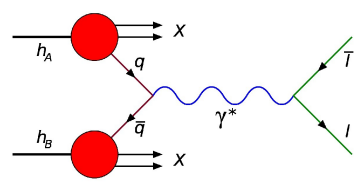

$\triangleright$ “ $q\left(x_{\text {beam }}\right) \bar{q}\left(x_{\text {target }}\right)$ " survives @ forward rapidity

$\triangleright q \mathrm{w} / x_{\text {beam }} \& \bar{q} \mathrm{w} / x_{\text {target }}$ are distinguishable event-by-event

- Proton \& deuteron as targets

$\triangleright$ Flavor separation $(\bar{u}$ vs $\bar{d}$ ) based on the proton-neutron isospin symmetry

$\triangleright$ Polarized $\mathrm{NH}_{3} \& \mathrm{ND}_{3}$ materials
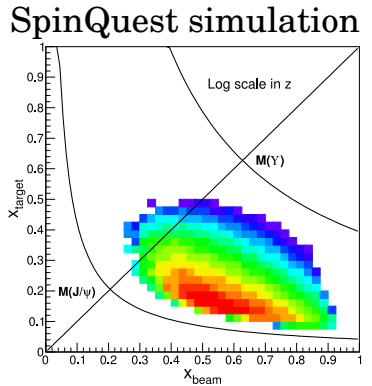


\section{Sivers Asymmetry in Drell-Yan Process}

- $p+N$ Drell-Yan process with transverse target polarization $\left(\vec{S}_{T}\right)$

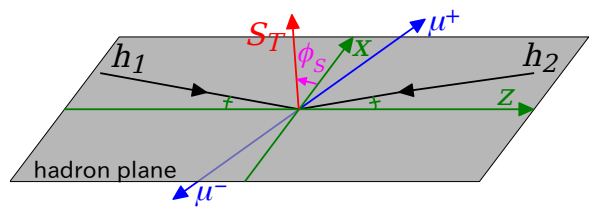

$\triangleright \phi_{S}$ : Azimuthal angle of target spin w.r.t. hadron plane

- $f_{1 T}^{\perp}$-related structure function

$$
\begin{aligned}
& \sigma \sim F_{U U}^{1}+S_{T} F_{U T}^{1} \sin \phi_{S} \\
& F_{U T}^{1}=-\mathcal{C}\left[\frac{\vec{q}_{T} \cdot \vec{k}_{T, \text { target }}}{q_{T} M_{p}} f_{1}\left(x_{\text {beam }}, \vec{k}_{T, \text { beam }}^{2}\right) f_{1 T}^{\perp}\left(x_{\text {target }}, \vec{k}_{T, \text { target }}^{2}\right)\right]
\end{aligned}
$$

$\triangleright \mathcal{C}$ : convolution over $\vec{k}_{T, \text { beam }} \& \vec{k}_{T, \text { target }}$ 
- Transverse Single Spin Asymmetry (TSSA): $A_{U T}^{\sin \phi_{S}}\left(x_{\text {target }}\right)$

$\triangleright N\left(x_{\text {target }}, \phi_{S}\right)$ : measured yields of Drell-Yan process

$\triangleright$ Fourier projection of $N$ on $\sin \phi_{S}$ modulation

$$
A_{U T}^{\sin \phi_{S}}\left(x_{\text {target }}\right) \equiv \frac{2}{f\left|S_{T}\right|} \frac{\int d \phi_{S} \frac{d N\left(x_{\text {target }}, \phi_{S}\right)}{d \phi_{S}} \sin \phi_{S}}{N\left(x_{\text {target }}\right)}=\frac{F_{U T}^{1}}{F_{U U}^{1}}
$$

$\triangleright \triangleright f$ : Dilution factor for the ratio of polarizable nucleons

$\triangleright$ Relation to $f_{1 T}^{\perp, \bar{q}}(x)$

$$
\frac{F_{U T}^{1}}{F_{U U}^{1}} \sim \frac{f\left(x_{\text {beam }}\right) \cdot f_{1 T}^{\perp, \bar{q}}\left(x_{\text {target }}\right)}{f\left(x_{\text {beam }}\right) \cdot \bar{f}\left(x_{\text {target }}\right)}
$$

$\triangle \triangleright$ Assumption for simplicity here:

quark having $x_{\text {beam }}$ at beam $\&$ anti-quark having $x_{\text {target }}$ at target

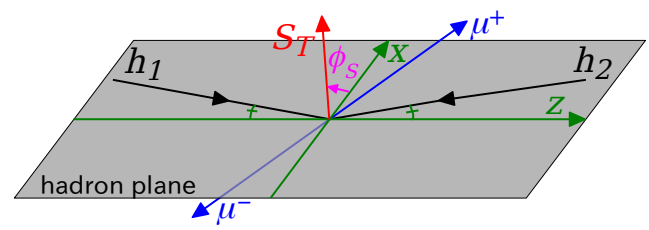




\section{Anticipated Sensitivity of E1039/SpinQuest}

- Conditions

$\triangleright$ Two years of data taking

$\triangleright \mathrm{NH}_{3}: \mathrm{ND}_{3}=50 \%: 50 \%$ in time

$\triangleright$ Details in the E1039 proposal

- TSSA: $A_{U T}^{\sin \phi_{S}}=A_{N}$

$\triangleright$ Measurement accuracy $\delta_{A_{N}} \sim 0.04$

$\triangleright$ Two predictions of $A_{N}$ of $\mathrm{NH}_{3}$

$\triangleright \triangleright$ Calculations based on SIDIS data

$\triangleright \triangleright$ Blue line takes into account the

Collins-Soper-Sterman scale evolution

- Aim to observe non-zero anti-quark Sivers asymmetry!!

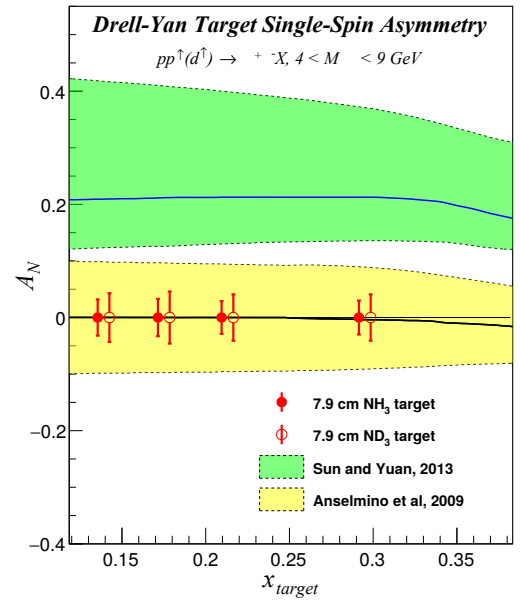

Phys. Rev. D88, 034016 (2013)

Eur. Phys. J. A39, 89 (2009) 


\section{Experimental Status}




\section{FNAL-E1039/SpinQuest Collaboration}

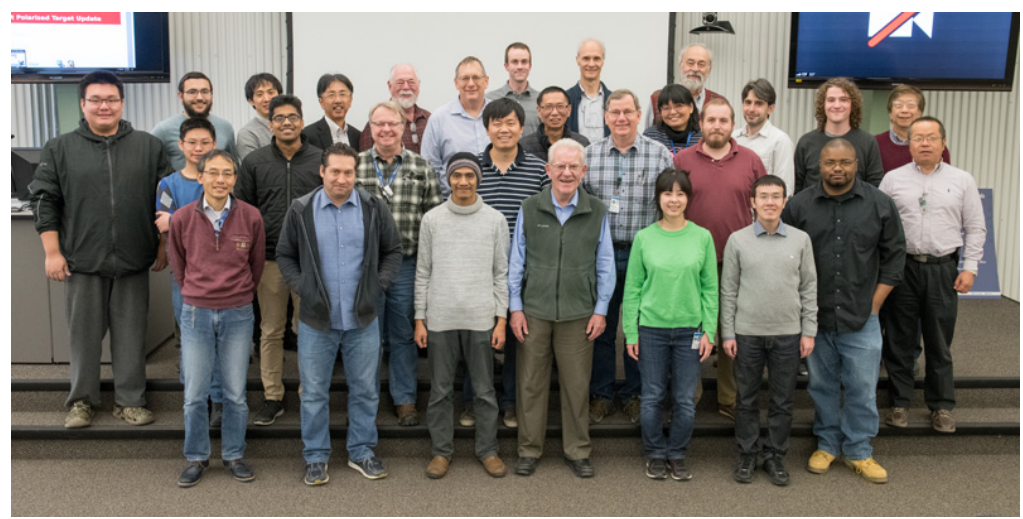

At Collaboration Meeting on March 7, 2019

- 15 institutions at present

- Spokespersons:

Kun Liu <liuk@fnal.gov> (LANL) \& Dustin Keller <dustin@ jlab.org> (UVA)

- https://spinquest.fnal.gov 


\section{E1039/SpinQuest Timeline}

\begin{tabular}{|c|c|c|}
\hline Year & Month & Event \\
\hline \multirow[t]{2}{*}{2018} & 05 & Granted Stage-2 approval from Fermilab \\
\hline & & Decommissioned E906/SeaQuest components \\
\hline \multirow[t]{5}{*}{2019} & 06 & Transfered the pol. target from UVA to Fermilab \\
\hline & Now & Commission all components using cosmic rays \\
\hline & Winter & Commission the whole system using proton beam \\
\hline & & Start the data taking \\
\hline & & $\Downarrow$ Run for two years \\
\hline 2021 & Winter & Finish the data taking \\
\hline
\end{tabular}

- We are considering quicker physics topics such as Transverse Single Spin Asymmetry (TSSA) of $J / \psi$ production

$\triangleright q+\bar{q} \gg g+g$ under E1039/SpinQuest kinematics

$\triangleright$ Sensitive to the Sivers function of anti-quarks and partly gluons 


\section{Fermilab Proton Beam}

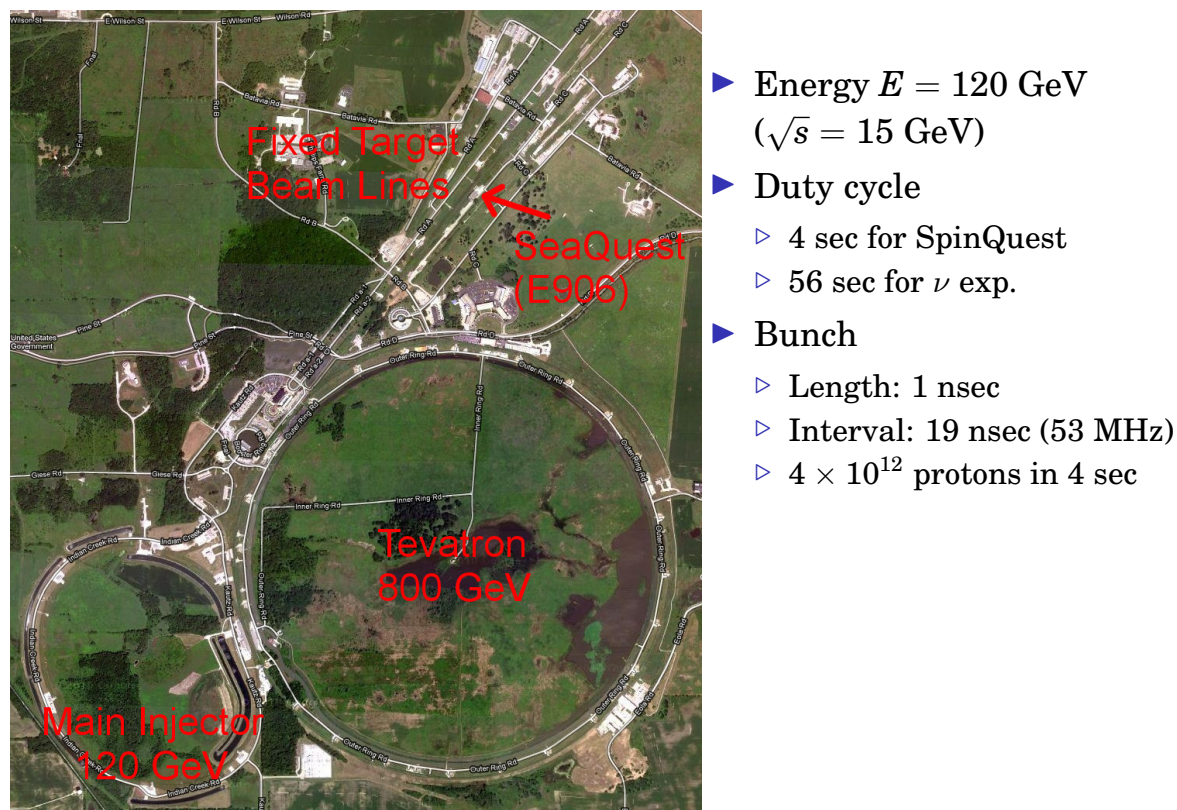




\section{E1039/SpinQuest Spectrometer}

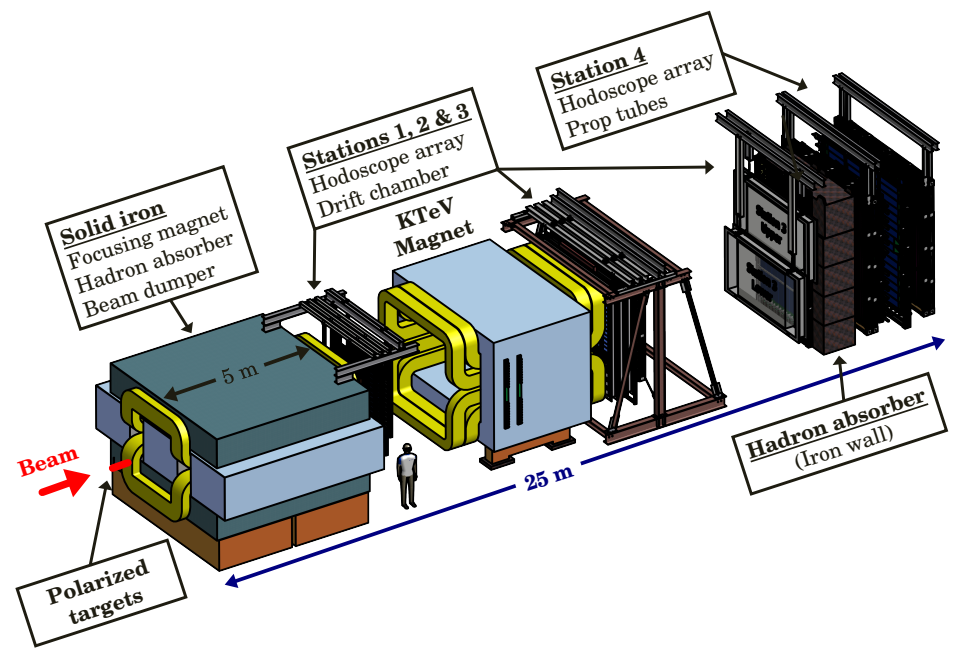

- Targets: transversely-polarized $\mathrm{NH}_{3} \& \mathrm{ND}_{3}$

- Focusing magnet (FMag) \& Tracking magnet (KMag)

- Iron inside FMag, as hadron absorber \& beam dump 
- Typical Drell-Yan event

$\triangleright$ Muon momenta $\sim 40 \mathrm{GeV} / c$

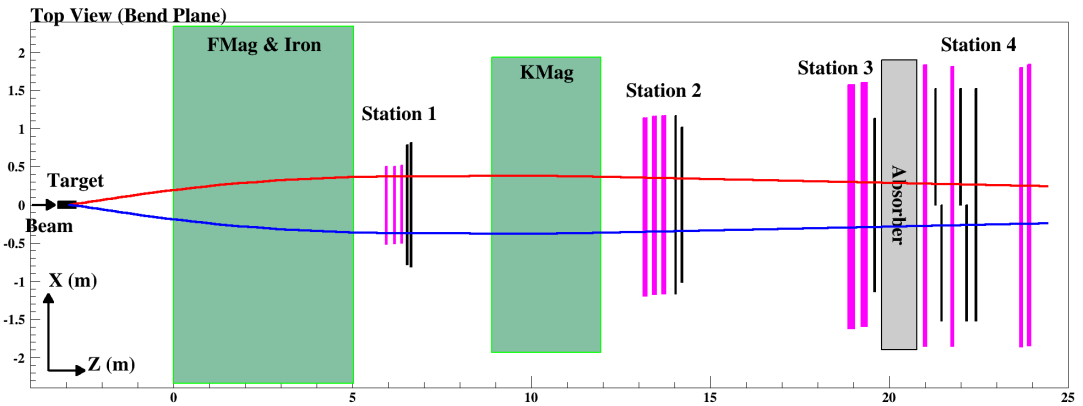

Detection of dimuons

$\triangleright$ Trigger with hodoscopes at Stations 1-4

$\triangleright \triangleright \times 7$-finer hodoscopes will be installed at Stations $1 \& 2$ (width $=1 \& 2 \mathrm{~cm}$ )

for better random-background rejection and dark-photon search

$\triangleright$ Tracking with drift chambers at Stations 1-3

$\triangleright$ Muon identification with drift tubes at Station 4

$\triangleright$ Resolution: $d M / M \lesssim 10 \%$ (dominated by the multiple scattering in FMag) 


\section{Beamline \& Shielding}

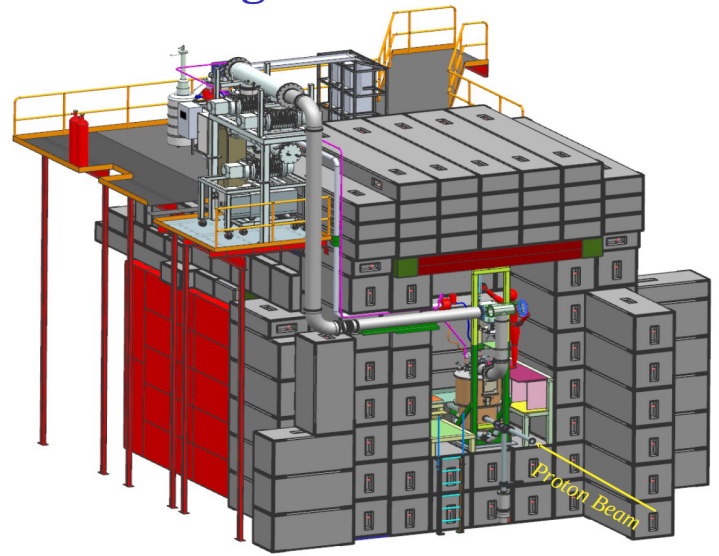

- Major modifications around target

$\triangleright$ More radiation shielding under new design

$\triangleright$ New cryo platform for target infrastructure

$\triangleright$ New location of target cave (300 cm upstream of FMag)

$\triangleright$ New collimator on beamline

- Special thanks to Fermilab accelerator division 
- NM3: looking downstream

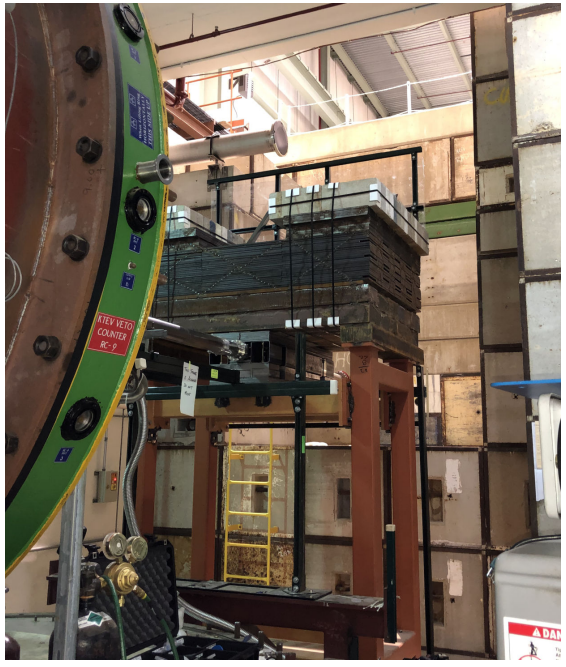

- NM4: looking upstream

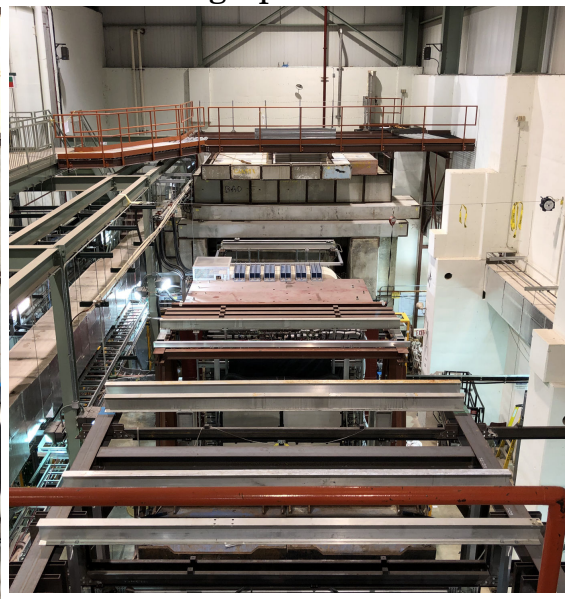

$\triangleright$ Beam collimator

$\triangleright$ Target cave

$\triangleright$ Rad. shielding

$\triangleright$ Cryo platform 


\section{Polarized Targets}

- Specification

$\triangleright$ Solid $\mathrm{NH}_{3} \& \mathrm{ND}_{3}$

$\triangleright$ Magnetic field: $B=5 \mathrm{~T}$ with $d B / B<10^{-4}$

$\triangleright$ Size: $L 80 \mathrm{~mm}, \phi 40 \mathrm{~mm}$

\begin{tabular}{c|ccccc}
\hline Material & Density & Dilution factor & Packing fraction & Polarization & Interaction length \\
\hline $\mathrm{NH}_{3}$ & $0.867 \mathrm{~g} / \mathrm{cm}^{3}$ & 0.176 & 0.60 & $80 \%$ & $5.3 \%$ \\
$\mathrm{ND}_{3}$ & $1.007 \mathrm{~g} / \mathrm{cm}^{3}$ & 0.300 & 0.60 & $32 \%$ & $5.7 \%$ \\
\hline
\end{tabular}

- New polarized-target construction ... massive efforts by LANL \& UVA

$\triangleright$ Change the field direction

$\triangleright$ Modify \& insert $1 \mathrm{~K}$ refrigerator

$\triangleright$ New device for cryostat

$\triangleright \triangleright$ LHe pumps for high cooling capacity

$\triangle \triangleright$ He liquefier for liquid helium recirculation

$\triangleright$ New device for polarization

$\triangleright \triangleright$ High power microwave source

$\triangleright \triangleright$ NMR system

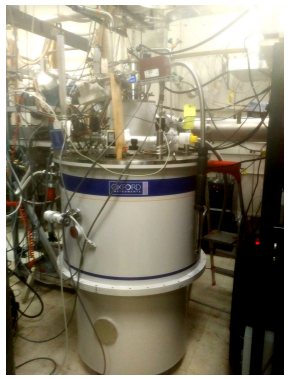


- Three material cells (+ one spare)

$\triangleright \mathrm{NH}_{3}, \mathrm{ND}_{3}$ \& Empty

$\triangleright$ Target materials will be replaced every $\sim 10$ days due to radiation damage

- Polarization

$\triangleright$ Via DNP

$\triangleright>90 \%$ for $\mathrm{NH}_{3}$

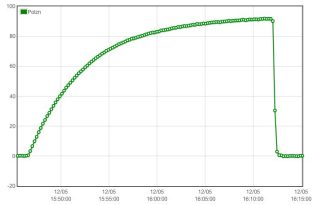

$\triangleright$ Flipped per day

- Performance under the beam radiation?

$\triangleright 4 \times 10^{12}$ protons in $4 \mathrm{sec}$

$\triangleright$ Feasible based on heat-deposit simulation

$\triangleright$ To be tested in the beam commissioning

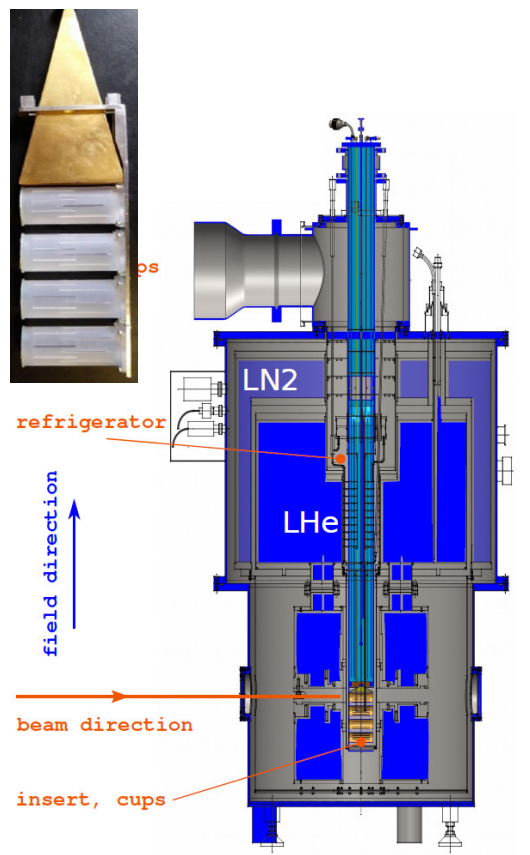




\section{Summary}

- The Sivers function of anti-quarks

$\triangleright$ Not well known, since not well separated from quark in SIDIS

$\triangleright$ Could be a "Smoking Gun" evidence for $L_{\bar{q}} \neq 0$

- E1039/SpinQuest at Fermilab

$\triangleright$ Measure TSSA of proton-induced Drell-Yan process

$\triangleright$ Use transversely-polarized $\mathrm{NH}_{3} \& \mathrm{ND}_{3}$ targets

$\triangleright$ Anticipate the measurement accuracy $\delta_{A_{N}} \sim 0.04$ at $0.1 \lesssim x \lesssim 0.3$

$\triangleright$ Extract the Sivers functions of $\bar{u} \& \bar{d}$ separately

- Preparation for the data taking

$\triangleright$ Commission all components using cosmic rays and then the proton beam

$\triangleright$ Start the data taking this winter for two years 